\title{
Weighted predictor-feedback formation control in local frames under time-varying delays and switching topology
}

\author{
Antonio González*, Rosario Aragüés, Gonzalo López-Nicolás, Carlos Sagüés * \\ Instituto de Investigación en Ingeniería de Aragón, Universidad de Zaragoza, María de Luna 1, 50018 Zaragoza, Spain
}

\begin{abstract}
This paper presents a novel control strategy based on predictor-feedback delay compensation for multiagent systems to reach a prescribed target formation under unknown but bounded communication delays and switching communication topology. Both communication delays and network topology can be subjected to arbitrarily-fast time variations. The key idea is to implement predictor-feedback strategies using only relative measurements between agents expressed in each local agent's frame, with the aim to counteract the negative effect of time delays. Nevertheless, due to the decentralized nature of the control, the presence of timevarying delays and switching communication topology, only partial delay compensation is possible. Despite this, we show that better performance can be achieved with our proposal with respect to non-predictor control schemes by introducing a weighting factor for predictor-feedback terms in the control law. Sufficient conditions based on Linear Matrix Inequalities (LMIs) for robust stability are also provided, which allow to easily design the controller parameters in order to maximize the speed of convergence. Finally, simulation results are provided to show the effectiveness of the proposed approach. Copyright (C) 2010 John Wiley \& Sons, Ltd.
\end{abstract}

Received ...

KEY WORDS: Delay compensation; Time-varying delay; Multi-agent system; Formation Control; Linear Matrix Inequality (LMI)

\section{INTRODUCTION}

Formation control for groups of autonomous mobile agents has received an increasing interest in the control community due to its high potential in different domains. A large variety of research areas involving formation control can be mentioned, for example: unmanned aerial vehicle (UAV) formation [?], autonomous multivehicle control [?], cooperative transport [?], etc. A key problem is how to design a distributed control strategy for the multiagent system to achieve a geometrical formation shape [?]. Different formation control strategies have been proposed depending on how the desired target formation is defined, including distance-based formation [?] or position-based formation in terms of absolute [?] and relative positions [?, ?].

It is worthwhile mentioning that a multiagent system constitutes a networked system, where the exchanged information between agents is often delayed due to the time elapsed during data transmission. Hence, time delays become a relevant issue in the stability analysis and synthesis of multiagent systems, since they may degrade the control-loop performance or even lead to instability $[?, ?]$. This fact has motivated the research of efficient and reliable methodology aimed at analyzing the impact of communication delays in closed-loop stability of multiagent systems [?, ?, ?]. For instance, the convergence of leader-follower synchronization of a network of agents has been studied in [?] by means of Lyapunov-Krasovskii functionals (LKF), including an estimator of time-varying delays in the control scheme to improve leader-tracking performance. In formation control, the stability analysis under time delays has been addressed for leader-based formation control strategies, 
such as formation-containment control [?, ?], tracking formation control [?] and multiple-leader based formation control with a stochastic sampling scheme in [?].

On the other hand, it is worthwhile mentioning that leaderless coordinate-free formation control strategies [?, ?, ?] can achieve global convergence to a unique rigid shape in the absence of a global coordinate system and leader agents, bringing more robustness and flexibility. For such coordinatefree formation control methods, the effect of time delays has also been investigated under timeconstant delays [?] and further extended to time-varying delays [?, ?], revealing that the speed of convergence is limited by the maximum allowable delay. Nevertheless, despite of the large number of contributions on this topic, little attention has been paid to investigate a possible improvement of the existing trade-off between speed of convergence and the maximum allowable time delay in multiagent formation control systems, which might prevent from reaching the target formation in a reasonable time interval if delays are large enough. This fact motivates the research of delay compensation methods applied to formation control synthesis.

The underlying idea behind delay compensation consists in obtaining a future prediction of the system state using past information with prediction horizon equivalent to the worst-case delay. Delay compensation requires prior knowledge of the system model in order to find an equivalent delay-free representation, and has the advantage of simplifying the control design [?, ?, ?]. Nevertheless, in distributed control systems, an exact future prediction of the system state cannot be obtained since the overall system model is not available to each agent. This fact, together with the presence of time-varying switching communication topology, makes the predictor-feedback control synthesis a non-trivial task.

Moreover, it is interesting to consider that only relative position measurements, expressed in each local agent's frame, are available. Under these premises, it is not necessary to share a global coordinate reference frame by all the agents. In this framework, different coordinate-free formation control synthesis algorithms were proposed to improve performance against time-varying delays for different communication topologies of the multiagent system: strongly connected communication topology [?], incomplete directed time-invariant topology [?], and time-varying switching topology [?]. Nevertheless, in all cases, the maximum value of the control gain $K$ (and therefore the speed of convergence) is limited by the worst-case delay. This limitation was overcome by applying delay compensation in coordinate-free formation control using multiple Smith predictors [?] with multiagent systems with a complete communication topology. Nevertheless, no conclusion was drawn about delay compensation control synthesis under time-varying delays and switching topology to improve closed-loop performance, which, to the best authors' knowledge, has not been investigated.

As contribution, we propose a novel predictor-feedback formation control strategy for systems with arbitrarily fast time-varying delays and switching topology, where the dynamics performance of the overall system can be improved by introducing a weighting factor for delay compensation terms in the predictor-feedback control. Through Lyapunov-Krasovskii approaches and small gain theory, sufficient conditions based on LMIs are provided in order to ascertain the exponential stability of the multiagent system with guaranteed decay rate performance, allowing to efficiently design the control gain and the weighting factor to maximize the speed of convergence.

\section{PROBLEM STATEMENT AND PRELIMINARIES}

Consider a multiagent system composed of $N$ agents, modeled as:

$$
\dot{q}_{i}(t)=u_{i}(t), \quad i=\{1, \ldots, N\},
$$

where $q_{i}(t)$ is the $2-D$ position vector of each agent, referred to any arbitrary reference frame. For each pair of agents $j, i \in\{1, \ldots, N\} \times\{1, \ldots, N\}, j \neq i$, we define $q_{j i}(t)=q_{j}(t)-q_{i}(t)$ and $c_{j i}$ respectively as the current and the desired relative position between them. The control action $u_{i}(t)$ must be designed to force the multiagent system (??) to reach a prescribed target formation, defined 
by the following set of interagent position vectors:

$$
c_{j i}=\left(c_{j i, x}, c_{j i, y}\right), \forall[j, i] \in\{1, \ldots, N\} \times\{1, \ldots, N\} .
$$

\section{Definition 1}

Let $p \geq 1$, and let $\left\{A_{1}, \cdots A_{p}\right\}$ be a finite collection of graphs. We define $\mathcal{A}_{\sigma(t)} \in\left\{A_{1}, \cdots A_{p}\right\}$ the time-varying adjacency matrix corresponding to a switching communication graph between all agents of system (??), where $\sigma(t) \in\{1, \ldots, p\}$ is a time-varying switching signal. Hence, $\mathcal{A}_{\sigma(t)}$ can be modeled as $\mathcal{A}_{\sigma(t)}=\sum_{s=1}^{p} \lambda_{s}(t) A_{s}$, where:

$$
\lambda_{s}(t)= \begin{cases}1, & \text { if } \sigma(t)=s \\ 0, & \text { otherwise }\end{cases}
$$

and

$$
A_{s}=\left[\begin{array}{cccc}
0 & a_{12}^{(s)} & \ldots & a_{1 N}^{(s)} \\
a_{21}^{(s)} & 0 & \cdots & a_{2 N}^{(s)} \\
\cdots & \cdots & \cdots & \cdots \\
a_{N 1}^{(s)} & a_{N 2}^{(s)} & \cdots & 0
\end{array}\right]
$$

where $a_{j i}^{(s)}=1$ if agent $i$ can receive information from agent $j$ at instant $t$, and $a_{j i}^{(s)}=0$ otherwise.

The following assumptions and remarks on multiagent system (??) are considered regarding the communication topology, the reference system available, and the nature of communication delays in order to define the problem to be addressed:

\section{Assumption 1}

Each adjacency matrix $A_{s}, s=1, \ldots, p$ defined in (??) corresponds to a directed communication topology, which contains at least a rooted directed spanning tree [?].

\section{Remark 1}

The adjacency matrix $\mathcal{A}_{\sigma(t)}$ given in Definition ?? is arbitrarily-fast time-varying, that is to say, the switching frequency is not necessarily limited.

\section{Remark 2}

The agents do not share a global reference frame.

\section{Remark 3}

The communication links between two agents $i$ and $j$ are affected by unknown arbitrarily-fast timevarying delays $\delta_{j i}(t)$ but bounded $\delta_{1} \leq \delta_{j i}(t) \leq \delta_{2}, \forall t \geq 0$, where $\delta_{1}, \delta_{2} \geq 0$ are known constants. Moreover, the functions $\delta_{j i}(t)$ are not necessarily symmetric $\delta_{j i}(t) \neq \delta_{i j}(t)$ and not necessarily continuous.

\section{PREDICTOR-FEEDBACK FORMATION CONTROL}

Let $t_{k}$ be the sampling instants $t_{k}=k T_{s}, k=0,1,2, \ldots$ with sampling period $T_{s}>0$. Hence, let us introduce the following time-triggered predictor-feedback formation control strategy for system (??):

$$
\begin{aligned}
& u_{i, k}=K \frac{1}{T_{s}} \sum_{j=1}^{N} a_{j i, k}\left(q_{j i, k-\tau_{j i, k}}-R\left(\hat{\phi}_{i, k}-\phi_{i}\right) c_{j i}\right) \\
& -\gamma K \frac{N}{2}\left(\sum_{f=1}^{\tau_{1}} u_{i, k-f}+\sum_{f=1}^{\tau_{2}} u_{i, k-f}\right),
\end{aligned}
$$


where $K>0$ is the control gain, $0 \leq \gamma \leq 1$ is a weighting factor for prediction terms (discussed later in Remark ??), $q_{j i, k-\tau_{j i, k}}$ are the available delayed relative position measurements to agent $i$, acquired at instants $t_{k}, k=0,1, \ldots$, and $a_{j i, k}=a_{j i}\left(t_{k}\right)=\sum_{i=1}^{p} \lambda_{s}\left(t_{k}\right) a_{j i}^{(s)}$, where $\lambda_{s}($.$) and a_{j i}^{(s)}$ are given in Definition ??. The function $R\left(\right.$.) denotes the $2-D$ rotation matrix, where $\phi_{i}$ is the rotation angle of each local agent's frame (assumed to be time-invariant), and $\hat{\phi}_{i, k}$ is the local rotation angle estimation, obtained by the following consensus law:

$$
\hat{\phi}_{i, k+1}=\hat{\phi}_{i, k}+v_{i, k}
$$

where

$$
\begin{gathered}
v_{i, k}=K \sum_{j=1}^{N} a_{j i, k}\left(\hat{\phi}_{j i, k-\tau_{j i, k}}-\phi_{j i}\right) \\
-\gamma K \frac{N}{2}\left(\sum_{f=1}^{\tau_{1}} v_{i, k-f}+\sum_{f=1}^{\tau_{2}} v_{i, k-f}\right),
\end{gathered}
$$

being $\phi_{j i}=\phi_{j}-\phi_{i}$ and $\hat{\phi}_{j i, k}=\hat{\phi}_{j, k}-\hat{\phi}_{i, k}$ the available measurements of the relative misalignment angles and their estimations, respectively.

\section{Remark 4}

The discrete-time delays $\tau_{1} \leq \tau_{j i, k} \leq \tau_{2}$ are obtained from the value rounded up to the next highest integer of the continuous-time delay $\delta_{1} \leq \delta_{j i}\left(t_{k}\right) \leq \delta_{2}$. leading to $\tau_{j i, k}=\operatorname{ceil}\left(\delta_{j i}\left(t_{k}\right) / T_{s}\right)$ with $\tau_{1}=\operatorname{ceil}\left(\delta_{1} / T_{s}\right), \tau_{2}=\operatorname{ceil}\left(\delta_{2} / T_{s}\right)$. Hence, the total delay will include the remaining time between the time instant in which a measurement is received, and the sampling instant $t_{k}$ in which the last received measurement is processed. This remaining time can be therefore expressed as $T_{s} \tau_{j i, k}-\delta_{j i}\left(t_{k}\right)$, which is bounded by the sampling period $T_{s}$. Hence, $T_{s}$ should be chosen sufficiently small with respect to communication delays in order to minimize the delay increment induced by sampling.

\section{Remark 5}

All relative position measurements $q_{j i, k}$ in (??) are expressed in each local agent's frame, whose orientation $\phi_{i}$ is not available to each agent due to the absence of a global shared reference frame (see Remark ??). To overcome this problem, we have introduced the consensus law (??)-(??) in order to find an agreement for a common reference frame, where the consensus parameters $K, \gamma$ must be designed to guarantee that $\lim _{k \rightarrow \infty} R\left(\varepsilon_{i, k}\right) c_{j i}=R(\alpha) c_{j i}$ in (??), being $\varepsilon_{i, k}=\hat{\phi}_{i, k}-\phi_{i}$ the estimation error of the rotation angle of each local agent's frame, and $\alpha$ the rotation angle of the agreed reference frame between all agents. The convergence analysis of (??)-(??), together with the formation control law (??), is later addressed in Remark ?? and Theorem ??. 


\subsection{Closed-loop dynamic equations}

The closed-loop system (??) with the proposed formation control (??) and (??) can be written in discrete-time form as:

$$
\begin{aligned}
& q_{i, k+1}=q_{i, k}+T_{s} u_{i, k} \\
& =q_{i, k}+K \sum_{j=1}^{N} a_{j i, k}\left(q_{j i, k-\tau_{j i, k}}-R\left(\varepsilon_{i, k}\right) c_{j i}\right) \\
& -\gamma K N \frac{T_{s}}{2}\left(\sum_{f=1}^{\tau_{1}} u_{i, k-f}+\sum_{f=1}^{\tau_{2}} u_{i, k-f}\right), \\
& \hat{\phi}_{i, k+1}=\hat{\phi}_{i, k}+v_{i, k} \\
& =\hat{\phi}_{i, k}+K \sum_{j=1}^{N} a_{j i, k}\left(\hat{\phi}_{j i, k-\tau_{j i, k}}-\phi_{j i}\right) \\
& -\gamma K \frac{N}{2}\left(\sum_{f=1}^{\tau_{1}} v_{i, k-f}+\sum_{f=1}^{\tau_{2}} v_{i, k-f}\right) .
\end{aligned}
$$

where $q_{i, k}$ are all agent's position vectors.Also, from (??) we have that $T_{s} u_{i, k}=q_{i, k+1}-q_{i, k}$ and $\hat{\phi}_{i, k+1}-\hat{\phi}_{i, k}=v_{i, k}$. Hence, we obtain the following equivalences:

$$
\begin{aligned}
& \frac{T_{s}}{2}\left(\sum_{f=1}^{\tau_{1}} u_{i, k-f}+\sum_{f=1}^{\tau_{2}} u_{i, k-f}\right)=q_{i, k}-\frac{1}{2} q_{i, k-\tau_{1}}-\frac{1}{2} q_{i, k-\tau_{2}}, \\
& \frac{1}{2}\left(\sum_{f=1}^{\tau_{1}} v_{i, k-f}+\sum_{f=1}^{\tau_{2}} v_{i, k-f}\right)=\hat{\phi}_{i, k}-\frac{1}{2} \hat{\phi}_{i, k-\tau_{1}}-\frac{1}{2} \hat{\phi}_{i, k-\tau_{2}}
\end{aligned}
$$

and the dynamic equations given in (??) can therefore be reformulated as:

$$
\begin{aligned}
& q_{i, k+1}=(1-\gamma K N) q_{i, k}+K \sum_{j=1}^{N} a_{j i, k}\left(q_{j i, k-\tau_{j i, k}}-R\left(\varepsilon_{i, k}\right) c_{j i}\right) \\
& +\gamma K \frac{N}{2}\left(q_{i, k-\tau_{1}}+q_{i, k-\tau_{2}}\right), \\
& \hat{\phi}_{i, k+1}=(1-\gamma K N) \hat{\phi}_{i, k}+K \sum_{j=1}^{N} a_{j i, k}\left(\hat{\phi}_{j i, k-\tau_{j i, k}}-\phi_{j i}\right) \\
& +\gamma K \frac{N}{2}\left(\hat{\phi}_{i, k-\tau_{1}}+\hat{\phi}_{i, k-\tau_{2}}\right) .
\end{aligned}
$$

Now, let us introduce the following definitions:

\section{Definition 2}

Given two agents $i$ and $j$, we define the relative formation error as:

$$
e_{j i, k}=q_{j i, k}-R(\alpha) c_{j i}
$$

where $\alpha$ is the agreement for the rotation angle of the reference frame, which must be reached by the consensus law (??)-(??) (see Remark ??).

\section{Definition 3}

Given two agents $i$ and $j$, we define the relative consensus error as:

$$
\varepsilon_{j i, k}=\varepsilon_{j, k}-\varepsilon_{i, k},
$$


or equivalently

$$
\varepsilon_{j i, k}=\hat{\phi}_{j i, k}-\phi_{j i},
$$

where $\varepsilon_{i, k}$ is introduced in Remark ??

With the above definitions and (??), we finally obtain the closed-loop system model expressed in terms of the relative errors $\varepsilon_{j i, k}$ and $e_{j i, k}$ :

$$
\begin{aligned}
& \varepsilon_{j i, k+1}=(1-\gamma K N) \varepsilon_{j i, k} \\
& +K \sum_{m=1}^{N}\left(a_{m j, k} \varepsilon_{m j, k-\tau_{m j, k}}-a_{m i, k} \varepsilon_{m i, k-\tau_{m i, k}}\right) \\
& +\gamma K \frac{N}{2}\left(\varepsilon_{j i, k-\tau_{1}}+\varepsilon_{j i, k-\tau_{2}}\right) \\
& e_{j i, k+1}=(1-\gamma K N) e_{j i, k} \\
& +K \sum_{m=1}^{N} a_{m j, k}\left(e_{m j, k-\tau_{m j, k}}+\omega_{m j, k}\right) \\
& -K \sum_{m=1}^{N} a_{m i, k}\left(e_{m i, k-\tau_{m i, k}}+\omega_{m i, k}\right) \\
& +\gamma K \frac{N}{2}\left(e_{j i, k-\tau_{1}}+e_{j i, k-\tau_{2}}\right)
\end{aligned}
$$

where

$$
\omega_{j i, k}=R(\alpha) c_{j i}-R\left(\varepsilon_{i, k}\right) c_{j i}
$$

\section{Remark 6}

On the one hand, by setting $\gamma=0$ in (??) and (??), we obtain the non-predictor formation control given in [?], where the maximum value of $K$ (and therefore the speed of convergence) is limited by the worst-case delay. On the other hand, by setting $\gamma=1$, the closed-loop system (??)-(??) renders delay-free in the presence of a strongly connected communication graph (that is to say, $a_{m j, k}=$ $a_{m i, k}=1, \forall j, i, i \neq j, \forall k \geq 0$ ) and known time-constant delays $\tau_{m j, k}=\tau_{m i, k}=\tau_{1}=\tau_{2} \equiv h$. In this case, the maximum speed of convergence can be achieved by setting $K=1 / N$, as can be deduced from the expressions:

$$
\begin{aligned}
& e_{j i, k+1}=(1-K N) e_{j i, k}+K \sum_{m=1}^{N}\left(\omega_{m j, k}-\omega_{m i, k}\right), \\
& \varepsilon_{j i, k+1}=(1-K N) \varepsilon_{j i, k},
\end{aligned}
$$

which are obtained from (??) and (??) by applying the equivalence $\sum_{m=1}^{N}\left(e_{m j, k}-e_{m i, k}\right)=$ $-N e_{j i, k}, \sum_{m=1}^{N}\left(\varepsilon_{m j, k}-\varepsilon_{m i, k}\right)=-N \varepsilon_{j i, k}$. Hence, the novelty introduced in the proposed formation control relies on the prediction terms introduced in the rightmost part of (??) and (??) weighted by the scalar factor $0 \leq \gamma \leq 1$, which offers an extra degree of freedom to improve closedloop performance in the control design. Moreover, it is worthwhile to recall that our predictor-based delay compensation is implemented by using only relative measurements expressed in each local agent's frame (decentralized predictor). As shown later, a suitable choice of the weighting factor $\gamma$ and the control gain $K$, may improve the closed-loop performance with respect to $\gamma=0$ (nonpredictor) and $\gamma=1$. Indeed, the proposed approach can be viewed as a partial delay compensation weighted by $\gamma$, whose benefits are later discussed.

\section{Definition 4}

Given a discrete-time system $x_{k+1}=f\left(x_{k}\right)$ with some initial condition $x_{0}$, we say that the system is $\beta$-stable if $x_{k}$ converges with some decay rate $0<\beta<1$, that is: $\left\|x_{k}\right\| \leq \mathcal{B}\left\|x_{0}\right\| \beta^{-k}, \forall k \geq 0$, for some $\mathcal{B}>0$. 
Control synthesis objective: Find the control parameters $K$ and $\gamma$ in order to maximize the speed of convergence (or minimize $\beta$ ) of the formation control system (??)-(??).

\section{STABILITY ANALYSIS}

This section addresses the robust $\beta$-stability analysis of the formation control system (??) and (??). To this end, we first obtain an equivalent interconnected representation for the closed-loop multiagent system (Section ??), which will be useful later to address the robust stability analysis (Section ??) and control synthesis (Remark ??) by small gain theory.

\subsection{An equivalent interconnected closed-loop system model}

The following lemma finds the equivalent closed-loop system model given in Fig. ?? for (??), (??) and (??), where the interconnected systems $S_{1}-\Delta_{1}$ and $S_{2}-\Delta_{2}$ correspond to (??) and (??) respectively, with

$$
\begin{aligned}
& \omega_{k}=\left(\omega_{21, k}, \omega_{31, k}, \omega_{41, k}, \cdots, \omega_{N 1, k},\right. \\
& \omega_{12, k}, \omega_{32, k}, \omega_{42, k}, \cdots, \omega_{N 2, k}, \\
& \cdots, \cdots, \cdots, \cdots, \\
& \left.\omega_{1 N, k}, \omega_{2 N, k}, \omega_{3 N, k}, \cdots, \omega_{N-1 N, k}\right)^{T},
\end{aligned}
$$

and $\omega_{j i, k}$ defined in (??). The forward subsystems $S_{1}, S_{2}$ are Linear Parameter Varying (LPV) systems with time-constant delays, and the feedback systems $\Delta_{1}$ and $\Delta_{2}$ are uncertain normbounded time-varying operators which contain all sources of time-varying delay mismatches.

\section{Lemma 1}

The closed-loop formation control system (??) and (??) is equivalent to the interconnected system given in Fig. ??, where:

$$
\begin{aligned}
& S_{1}:\left\{\begin{array}{l}
\varepsilon_{k+1}=(1-\gamma K N) \varepsilon_{k} \\
+\left(T^{+} \mathcal{G}_{k} T\right)\left(\varepsilon_{k-\tau_{1}}+\varepsilon_{k-\tau_{2}}\right)+\tau\left(T^{+} \mathcal{H}_{k}\right) \rho_{k}, \\
y_{k}^{\rho}=-\gamma K N T \varepsilon_{k}+\left(\mathcal{G}_{k} T\right)\left(\varepsilon_{k-\tau_{1}}+\varepsilon_{k-\tau_{2}}\right)+\tau \mathcal{H}_{k} \rho_{k},
\end{array}\right. \\
& \Delta_{1}: \rho_{k}=\Delta_{1, k} y_{k}^{\rho},
\end{aligned}
$$

where

$$
\begin{aligned}
\varepsilon_{k} & =\left(\begin{array}{lll}
\varepsilon_{21, k}, & \cdots, & \varepsilon_{N 1, k}
\end{array}\right)^{T} \in \mathcal{R}^{N-1}, \\
e_{k} & =\left(\begin{array}{lll}
e_{21, k}, & \cdots, & e_{N 1, k}
\end{array}\right)^{T} \in \mathcal{R}^{2(N-1)},
\end{aligned}
$$




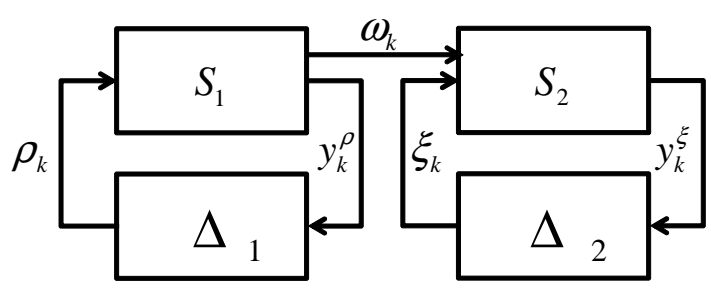

Figure 1. Interconnected system model for the multiagent system (??) with (??), (??) and (??).

and $\omega_{k}$ is defined in (??). $\Delta_{1, k} \in \mathcal{R}^{\bar{N}}$ and $\Delta_{2, k} \in \mathcal{R}^{2 \bar{N}}$ with $\bar{N}=N(N-1)$ are unitary normbounded time-varying operators with diagonal structure: $\left\|\Delta_{1, k}\right\|_{\infty} \leq 1,\left\|\Delta_{2, k}\right\|_{\infty} \leq 1$, and

$$
\begin{aligned}
& \tau=\tau_{2}-\tau_{1}, \quad \bar{N}=N(N-1) \\
& \mathcal{G}_{k}=\frac{K}{2}\left(\gamma N \cdot I_{\bar{N}}+\mathcal{F}_{k}\right), \quad \mathcal{H}_{k}=\frac{K}{2} \mathcal{F}_{k}, \\
& \mathcal{F}_{k}=\sum_{s=1}^{p} \lambda_{s, k} F_{s}, \quad F_{s}=\left(T_{1}-T_{2}\right) \otimes \mathbf{1}_{1 \times(N-1)} \cdot \operatorname{diag}\left(\bar{a}_{s}\right), \\
& T^{+}=\left[\begin{array}{lll}
I_{N-1} & \mathbf{0}_{(N-1) \times(\bar{N}-N+1)}
\end{array}\right], \quad T=\left(T_{1}-T_{2}\right)\left[\begin{array}{c}
\mathbf{0}_{1 \times(N-1)} \\
I_{N-1}
\end{array}\right], \\
& T_{1}=\left[\begin{array}{lll}
\left(\mathcal{I}_{N}^{1}\right)^{T} & \ldots & \left(\mathcal{I}_{N}^{N}\right)^{T}
\end{array}\right]^{T}, \quad T_{2}=I_{N} \otimes \mathbf{1}_{(N-1) \times 1}
\end{aligned}
$$

where $\bar{a}_{s}$ gathers all the entries of (??), arranged as:

$$
\begin{aligned}
& \bar{a}_{s}=\left(a_{21}^{(s)}, a_{31}^{(s)}, a_{41}^{(s)}, \cdots, a_{N 1}^{(s)},\right. \\
& a_{12}^{(s)}, a_{32}^{(s)}, a_{42}^{(s)}, \cdots, a_{N 2}^{(s)}, \\
& \cdots, \cdots, \cdots, \cdots, \\
& \left.a_{1 N}^{(s)}, a_{2 N}^{(s)}, a_{3 N}^{(s)}, \cdots, a_{N-1 N}^{(s)}\right)^{T} .
\end{aligned}
$$

and the scalar functions $\lambda_{s, k}=\left.\lambda_{s}(t)\right|_{t=k T_{s}}$ with $\lambda_{s}(t)$ are defined in (??). The symbol $\otimes$ stands for the Kronecker product, and the symbols $\mathcal{I}_{N}^{r}, 1 \leq r \leq N$ stand for matrices of dimension $(N-1) \times N$ obtained from the identity matrix $I_{N}$ by removing its $r_{t h}$ row.

Proof

Let us obtain separately the subsystem models $S_{1}-\Delta_{1}$ and $S_{2}-\Delta_{2}$ :

Subsystem $S_{1}-\Delta_{1}$ : First, let us write $\varepsilon_{j i, k-\tau_{j i, k}}$ as

$$
\varepsilon_{j i, k-\tau_{j i, k}}=\frac{1}{2}\left(\varepsilon_{j i, k-\tau_{1}}+\varepsilon_{j i, k-\tau_{2}}+\tau \rho_{j i, k}\right)
$$

where $\tau=\tau_{2}-\tau_{1}$ and

$$
\rho_{j i, k}=\frac{2}{\tau}\left(\varepsilon_{j i, k-\tau_{j i, k}}-\frac{1}{2}\left(\varepsilon_{j i, k-\tau_{1}}+\varepsilon_{j i, k-\tau_{2}}\right)\right) .
$$


Applying (??), system (??) can be rewritten as:

$$
\begin{aligned}
& \varepsilon_{j i, k+1}=(1-\gamma K N) \varepsilon_{j i, k} \\
& +\frac{K}{2}\left(\gamma N \varepsilon_{j i, k-\tau_{1}}+\sum_{m=1}^{N}\left(a_{m j, k} \varepsilon_{m j, k-\tau_{1}}-a_{m i, k} \varepsilon_{m i, k-\tau_{1}}\right)\right) \\
& +\frac{K}{2}\left(\gamma N \varepsilon_{j i, k-\tau_{2}}+\sum_{m=1}^{N}\left(a_{m j, k} \varepsilon_{m j, k-\tau_{2}}-a_{m i, k} \varepsilon_{m i, k-\tau_{2}}\right)\right) \\
& +\frac{\tau K}{2}\left(\sum_{m=1}^{N} a_{m j, k} \rho_{m j, k}-\sum_{m=1}^{N} a_{m i, k} \rho_{m i, k}\right) .
\end{aligned}
$$

By applying Lemma ?? (see Appendix), it can be deduced that there exists a time-varying operator $\Delta_{j i, k}^{(1)} \in \mathcal{R}$ satisfying $\left\|\Delta_{j i, k}^{(1)}\right\|_{\infty} \leq 1$ and:

$$
\rho_{j i, k}=\Delta_{j i, k}^{(1)} y_{j i, k}^{\rho},
$$

where $\rho_{j i, k}$ is the input defined in (??), and

$$
y_{j i, k}^{\rho}=\varepsilon_{j i, k+1}-\varepsilon_{j i, k}
$$

Note the equivalence $\sum_{m=1}^{N}\left(a_{m j, k} \varepsilon_{m j, k}-a_{m i, k} \varepsilon_{m i, k}\right)=\mathcal{F}_{k} \bar{\varepsilon}_{k}$, with $\mathcal{F}_{k}$ given in (??) and $\bar{\varepsilon}_{k}$ given below in (??). Hence, all the dynamic equations (??), (??) and (??) can be put in matricial form as:

$$
\begin{aligned}
& \bar{\varepsilon}_{k+1}=(1-\gamma K N) \bar{\varepsilon}_{k}+\mathcal{G}_{k} \bar{\varepsilon}_{k-\tau_{1}}+\mathcal{G}_{k} \bar{\varepsilon}_{k-\tau_{2}}+\tau \mathcal{H}_{k} \rho_{k}, \\
& \rho_{k}=\Delta_{1, k} y_{k}^{\rho}, \quad \Delta_{1, k}=\operatorname{diag}\left(\Delta_{21, k}^{(1)}, \cdots, \Delta_{N-1 N, k}^{(1)}\right) \\
& y_{k}^{\rho}=-\gamma K N \bar{\varepsilon}_{k}+\mathcal{G}_{k} \bar{\varepsilon}_{k-\tau_{1}}+\mathcal{G}_{k} \bar{\varepsilon}_{k-\tau_{2}}+\tau \mathcal{H}_{k} \rho_{k},
\end{aligned}
$$

where $\tau, \mathcal{G}_{k}, \mathcal{H}_{k}$ are defined in (??), and

$$
\begin{aligned}
\bar{\varepsilon}_{k} & =\left[\varepsilon_{21, k}, \cdots, \varepsilon_{N-1 N, k}\right]^{T} \in \mathcal{R}^{N(N-1)}, \\
\rho_{k} & =\left[\rho_{21, k}, \cdots, \rho_{N-1 N, k}\right]^{T} \in \mathcal{R}^{N(N-1)}, \\
y_{k}^{\rho} & =\left[y_{21, k}^{\rho}, \cdots, y_{N-1 N, k}^{\rho}\right]^{T} \in \mathcal{R}^{N(N-1)}
\end{aligned}
$$

are augmented vectors containing respectively all terms $\varepsilon_{j i, k}, \rho_{j i, k}, y_{j i, k}^{\rho}$, arranged in a similar fashion as $\omega_{k}$ given in (??).

Note that all the relative errors $e_{j i}, \varepsilon_{j i}, j \neq i$ can be expressed as a function of $e_{j 1}, \varepsilon_{j 1}, j=$ $2, \ldots, N$. Hence, (??) and (??) can equivalently be modeled as the interconnected subsystem $S_{1}-\Delta_{1}$ by applying the state transformations $\varepsilon_{k}=T^{+} \bar{\varepsilon}_{k}, \bar{\varepsilon}_{k}=T \varepsilon_{k}$ and $\rho_{k}=\Delta_{1, k} y_{\rho_{k}}$.

Subsystem $S_{2}-\Delta_{2}$ : Analogously, let us write $e_{j i, k-\tau_{j i, k}}$ as

$$
e_{j i, k-\tau_{j i, k}}=\frac{1}{2}\left(e_{j i, k-\tau_{1}}+e_{j i, k-\tau_{2}}+\tau \xi_{j i, k}\right)
$$

where $\tau=\tau_{2}-\tau_{1}$ and

$$
\xi_{j i, k}=\frac{2}{\tau}\left(e_{j i, k-\tau_{j i, k}}-\frac{1}{2}\left(e_{j i, k-\tau_{1}}+e_{j i, k-\tau_{2}}\right)\right) .
$$


Applying (??), system (??) can be rewritten as:

$$
\begin{aligned}
& e_{j i, k+1}=(1-\gamma K N) e_{j i, k} \\
& +\frac{K}{2}\left(\gamma N e_{j i, k-\tau_{1}}+\sum_{m=1}^{N}\left(a_{m j, k} e_{m j, k-\tau_{1}}-a_{m i, k} e_{m i, k-\tau_{1}}\right)\right) \\
& +\frac{K}{2}\left(\gamma N e_{j i, k-\tau_{2}}+\sum_{m=1}^{N}\left(a_{m j, k} e_{m j, k-\tau_{2}}-a_{m i, k} e_{m i, k-\tau_{2}}\right)\right) \\
& +\frac{\tau K}{2}\left(\sum_{m=1}^{N} a_{m j, k} \xi_{m j, k}-\sum_{m=1}^{N} a_{m i, k} \xi_{m i, k}\right) \\
& +K\left(\sum_{m=1}^{N} a_{m j, k} \omega_{m j, k}-\sum_{m=1}^{N} a_{m i, k} \omega_{m i, k}\right) .
\end{aligned}
$$

By applying Lemma ?? (see Appendix), it can be deduced that there exists a time-varying operator $\Delta_{j i, k}^{(2)} \in \mathcal{R}^{2}$ satisfying $\left\|\Delta_{j i, k}^{(2)}\right\|_{\infty} \leq 1$, and:

$$
\xi_{j i, k}=\Delta_{j i, k}^{(2)} y_{j i, k}^{\xi}
$$

where

$$
y_{j i, k}^{\xi}=e_{j i, k+1}-e_{j i, k} .
$$

The rest of the proof can be completed following the same steps as for subsystem $S_{1}-\Delta_{1}$ by taking into account $\omega_{k}$ in (??), and defining

$$
\begin{aligned}
& \bar{e}_{k}=\left[e_{21, k}, \cdots, e_{N-1 N, k}\right]^{T} \in \mathcal{R}^{2 N(N-1)}, \\
& \xi_{k}=\left[\xi_{21, k}, \cdots, \xi_{N-1 N, k}\right]^{T} \in \mathcal{R}^{2 N(N-1)}, \\
& y_{k}^{\xi}=\left[y_{21, k}^{\xi}, \cdots, y_{N-1 N, k}^{\xi}\right]^{T} \in \mathcal{R}^{2 N(N-1)}, \\
& \Delta_{2, k}=\operatorname{diag}\left(\Delta_{21, k}^{(2)}, \cdots, \Delta_{N-1 N, k}^{(2)}\right),
\end{aligned}
$$

and applying the state transformations $e_{k}=\left(T^{+} \otimes I_{2}\right) \bar{e}_{k}, \bar{e}_{k}=\left(T \otimes I_{2}\right) e_{k}$.

\section{Remark 7}

Note that $\varepsilon_{i, k}=\hat{\phi}_{i, k}-\phi_{i}$ converges to the agreed rotation angle $\alpha$ if and only if subsystem $S_{1}-\Delta_{1}$ is robustly stable for any time-varying delay and switching topology. In this case, all the entries $\omega_{j i, k}$ given in (??) vanish, as long as $k \rightarrow \infty$, and the term $R\left(\hat{\phi}_{i, k}-\phi_{i}\right) c_{j i}$ in (??) converges to the rotated reference frame $R(\alpha) c_{j i}$. Hence, the stability of the overall system (??) can be ascertained by proving the stability of $S_{1}-\Delta_{1}$ and $S_{2}-\Delta_{2}$ separately.

As pointed out in Remark ??, only partial delay compensation might be achievable due to the presence of time-varying delay mismatches and switching topology in the decentralized setup considered here. Next sections address the stability analysis and control design (parameters $K$ and $\gamma$ ) to improve closed-loop performance in terms of speed of convergence.

\subsection{Sufficient LMI conditions for stability analysis}

This section gives sufficient conditions based on LMIs for the $\beta$-stability of the multiagent system (??) with the proposed predictor-based formation control (??). Note that the equivalent system model given in Lemma ?? (see Fig. ??) is composed of two systems: $\left(S_{1}-\Delta_{1}\right)$ and $\left(S_{2}-\Delta_{2}\right)$ respectively. In accordance with Remark ??, the $\beta$ - stability of the overall system can be demonstrated by proving separately that the two interconnected systems $\left(S_{1}-\Delta_{1}\right)$ and $\left(S_{2}-\Delta_{2}\right)$ are stable, as explained in the following theorem: 


\section{Theorem 1}

Given the controller parameters $0 \leq \gamma \leq 1, K>0$, the lower and upper bound delays $\tau_{1}, \tau_{2}$, and the decay rate $\beta$, system (??) with control law (??) is $\beta$-stable if there exist symmetric matrices $P, Q_{1}, Q_{2}, Z_{1}, Z_{2} \in \mathcal{R}^{N-1}>0$ and positive scalars $v_{1}, v_{2}, \cdots, v_{\bar{N}}$ such that the following LMIs hold, $\forall s \in[1, \cdots, p]$ :

$$
\Pi_{s}=\left[\begin{array}{cccc}
\Pi & \Omega_{1, s}^{T} P & \Omega_{2, s}^{T} Z & \Omega_{3, s}^{T} W \\
(*) & -P & 0 & 0 \\
(*) & (*) & -Z & 0 \\
(*) & (*) & (*) & -W
\end{array}\right]<0
$$

where

$$
\begin{aligned}
& \Pi=\left[\begin{array}{cccc}
\Pi_{11} & \Pi_{12} & \Pi_{13} & 0 \\
(*) & \Pi_{22} & 0 & 0 \\
(*) & (*) & \Pi_{33} & 0 \\
(*) & (*) & (*) & -W
\end{array}\right], \Omega_{1, s}^{T}=\left[\begin{array}{c}
\Omega^{T} \\
\left(T^{+} G_{s} T\right)^{T} \\
\left(T^{+} G_{s} T\right)^{T} \\
\tau\left(T^{+} H_{s}\right)^{T}
\end{array}\right], \\
& \Omega_{2, s}^{T}=\left[\begin{array}{c}
\Omega^{T}-I_{N-1} \\
\left(T^{+} G_{s} T\right)^{T} \\
\left(T^{+} G_{s} T\right)^{T} \\
\tau\left(T^{+} H_{s}\right)^{T}
\end{array}\right], \Omega_{3, s}^{T}=\left[\begin{array}{c}
-\gamma K T^{T} \\
\left(G_{s} T\right)^{T} \\
\left(G_{s} T\right)^{T} \\
\tau H_{s}^{T}
\end{array}\right] \\
& \Omega=(1-\gamma K N) \cdot I_{N-1}, \\
& G_{s}=\frac{K}{2}\left(\gamma N \cdot I_{\bar{N}}+F_{s}\right), \quad H_{s}=\frac{K}{2} F_{s}, \\
& \Pi_{11}=-\beta^{2} P+Q_{1}+Q_{2}-\beta^{2 \tau_{1}} Z_{1}-\beta^{2 \tau_{2}} Z_{2}, \\
& \Pi_{12}=\beta^{2 \tau_{1}} Z_{1}, \\
& \Pi_{13}=\beta^{2 \tau_{2}} Z_{2}, \\
& \Pi_{22}=-\beta^{2 \tau_{1}} Q_{1}-\beta^{2 \tau_{1}} Z_{1}, \\
& \Pi_{33}=-\beta^{2 \tau_{2}} Q_{2}-\beta^{2 \tau_{2}} Z_{2}, \\
& Z=\tau_{1} \mu_{1} Z_{1}+\tau_{2} \mu_{2} Z_{2}, \quad W=\operatorname{diag}\left(v_{1}, v_{2}, \cdots, v_{\bar{N}}\right), \\
& \mu_{1}=\sum_{f=1}^{\tau_{1}} \beta^{2(f-1)}, \quad \mu_{2}=\sum_{f=1}^{\tau_{2}} \beta^{2(f-1)},
\end{aligned}
$$

and $\tau, F_{s}, T, T^{+}$defined in Lemma (??).

\section{Proof}

From the equivalence given in Lemma ??, the convergence of the formation control can be proved by checking the robust stability of system (??). First, let us go with system $S_{1}-\Delta_{1}$ : Stability of $S_{1}-\Delta_{1}$ : Consider the following Lyapunov-Krasovskii functional:

$$
V_{k}=V_{1, k}+V_{2, k}+V_{3, k},
$$

where

$$
\begin{aligned}
V_{1, k} & =\varepsilon_{k}^{T} P \varepsilon_{k}, \\
V_{2, k} & =\sum_{m=1}^{\tau_{1}} \beta^{2(m-1)} \varepsilon_{k-m}^{T} Q_{1} \varepsilon_{k-m}+\sum_{m=1}^{\tau_{2}} \beta^{2(m-1)} \varepsilon_{k-m}^{T} Q_{2} \varepsilon_{k-m}, \\
V_{3, k} & =\tau_{1} \sum_{f=1}^{\tau_{1}} \sum_{m=1}^{f} \beta^{2(f-1)} \eta_{k-m}^{T} Z_{1} \eta_{k-m}+\tau_{2} \sum_{f=1}^{\tau_{2}} \sum_{m=1}^{f} \beta^{2(f-1)} \eta_{k-m}^{T} Z_{2} \eta_{k-m}
\end{aligned}
$$


and $\eta_{k}=\varepsilon_{k+1}-\varepsilon_{k}$. Taking into account system $S_{1}$ in (??), the increments $\Delta_{i, k}^{V}=V_{i, k+1}-$ $\beta^{2} V_{i, k}, i=1,2,3$ yield respectively:

$$
\begin{aligned}
& \Delta_{1, k}^{V}=\varepsilon_{k+1}^{T} P \varepsilon_{k+1}-\beta^{2} \varepsilon_{k}^{T} P \varepsilon_{k} \\
& =\chi_{k}^{T}\left(\Xi_{1}+\Omega_{1, k}^{T} P \Omega_{1, k}\right) \chi_{k}, \\
& \Delta_{2, k}^{V}=\varepsilon_{k}^{T}\left(Q_{1}+Q_{2}\right) \varepsilon_{k}-\beta^{2 \tau_{1}} \varepsilon_{k-\tau_{1}}^{T} Q_{1} \varepsilon_{k-\tau_{1}}-\beta^{2 \tau_{2}} \varepsilon_{k-\tau_{2}}^{T} Q_{2} \varepsilon_{k-\tau_{2}} \\
& =\chi_{k}^{T} \Xi_{2} \chi_{k}, \\
& \Delta_{3, k}^{V}=\left(\tau_{1} \mu_{1}\right) \eta_{k}^{T} Z_{1} \eta_{k}+\left(\tau_{2} \mu_{2}\right) \eta_{k}^{T} Z_{2} \eta_{k} \\
& -\beta^{2 \tau_{1}}\left(\tau_{1} \sum_{m=k-\tau_{1}}^{k-1} \eta_{m}^{T} Z_{1} \eta_{m}\right)-\beta^{2 \tau_{2}}\left(\tau_{2} \sum_{m=k-\tau_{2}}^{k-1} \eta_{m}^{T} Z_{2} \eta_{m}\right),
\end{aligned}
$$

and

$$
\begin{aligned}
& \Xi_{1}=\operatorname{diag}\left(-\beta^{2} P, \quad 0, \quad 0, \quad 0\right), \\
& \Xi_{2}=\operatorname{diag}\left(Q_{1}+Q_{2}, \quad-\beta^{2 \tau_{1}} Q_{1}, \quad-\beta^{2 \tau_{2}} Q_{2}, \quad 0\right),
\end{aligned}
$$

where $\Omega_{i, k}=\sum_{s=1}^{p} \lambda_{s, k} \Omega_{i, s}, i=1,2,3$ with $\lambda_{s, k}=\lambda\left(k T_{s}\right)$ defined in (??), $\Omega_{i, s}$ defined in (??), and $\chi_{k}=\left[\begin{array}{cccc}\varepsilon_{k}^{T} & \varepsilon_{k-\tau_{1}}^{T} & \varepsilon_{k-\tau_{2}}^{T} & \rho_{k}^{T}\end{array}\right]^{T}$. Applying Jensen's inequality [?], we have that

$$
\begin{aligned}
& -\tau_{1} \sum_{m=k-\tau_{1}}^{k-1} \eta_{m}^{T} Z_{1} \eta_{m} \leq\left(\sum_{m=k-\tau_{1}}^{k-1} \eta_{m}^{T}\right) Z_{1}\left(\sum_{m=k-\tau_{1}}^{k-1} \eta_{m}\right) \\
& =\left(\varepsilon_{k}-\varepsilon_{k-\tau_{1}}\right)^{T} Z_{1}\left(\varepsilon_{k}-\varepsilon_{k-\tau_{1}}\right), \\
& -\tau_{2} \sum_{m=k-\tau_{2}}^{k-1} \eta_{m}^{T} Z_{2} \eta_{m} \leq\left(\sum_{m=k-\tau_{2}}^{k-1} \eta_{m}^{T}\right) Z_{2}\left(\sum_{m=k-\tau_{2}}^{k-1} \eta_{m}\right) \\
& =\left(\varepsilon_{k}-\varepsilon_{k-\tau_{2}}\right)^{T} Z_{2}\left(\varepsilon_{k}-\varepsilon_{k-\tau_{2}}\right) .
\end{aligned}
$$

Also, recalling that $Z=\tau_{1} \mu_{1} Z_{1}+\tau_{2} \mu_{2} Z_{2}, \eta_{k}=\varepsilon_{k+1}-\varepsilon_{k}$, and $\Omega_{2, k}=\sum_{s=1}^{p} \lambda_{s, k} \Omega_{2, s}, i=1,2,3$ with $\Omega_{2, s}$ defined in (??), we obtain the following equivalence:

$$
\tau_{1} \mu_{1} \eta_{k}^{T} Z_{1} \eta_{k}+\tau_{2} \mu_{2} \eta_{k}^{T} Z_{2} \eta_{k}=\chi_{k}^{T}\left(\Omega_{2, k}^{T} Z \Omega_{2, k}\right) \chi_{k}
$$

Replacing (??) and (??) into $\Delta_{3, k}^{V}$ in (??) we obtain:

$$
\Delta_{3, k}^{V} \leq \chi_{k}^{T}\left(\Omega_{2, k}^{T} Z \Omega_{2, k}\right) \chi_{k}+\chi_{k}^{T} \Xi_{3} \chi_{k}^{T},
$$

where

$$
\Xi_{3}=\left[\begin{array}{cccc}
-\beta^{2 \tau_{1}} Z_{1}-\beta^{2 \tau_{2}} Z_{2} & \Pi_{12} & \Pi_{13} & 0 \\
(*) & -\beta^{2 \tau_{1}} Z_{1} & 0 & 0 \\
(*) & (*) & -\beta^{2 \tau_{1}} Z_{2} & 0 \\
(*) & (*) & (*) & 0
\end{array}\right] .
$$

Noting that $W>0$ defined in (??) is of diagonal structure, there exists a diagonal matrix $\mathcal{X}=\operatorname{diag}\left(x_{1}, \ldots, x_{\bar{N}}\right)$ for some scalars $x_{i} \neq 0, i=1, \ldots, \bar{N}$ such that $W=\mathcal{X}^{2}$ and $\mathcal{X} \Delta_{1, k}=$ $\Delta_{1, k} \mathcal{X}, \forall k \geq 0$, taking into account the diagonal structure of the time-varying operator $\Delta_{1, k}$ given in (??). To prove the stability of the interconnected system $S_{1}-\Delta_{1}$, it suffices to prove that the fulfilment of (??) implies the two conditions given in Theorem ??: (i) the internal $\beta$-stability of system $S_{1}$, and (ii) the condition $\left\|\mathcal{X} S_{1} \mathcal{X}^{-1}\right\|_{\infty}<1$. Both conditions can be ensured by proving the existence of a diagonal matrix $W>0$ and a Lyapunov-Krasovskii functional (??) such that the 
following inequality is true:

$$
\Delta_{k}^{V}+\left(y_{k}^{\rho}\right)^{T} W y_{k}^{\rho}-\rho_{k}^{T} W \rho_{k}<0,
$$

where $\Delta_{k}^{V}=\Delta_{1, k}^{V}+\Delta_{2, k}^{V}+\Delta_{3, k}^{V}$. From (??) and taking into account that $y_{k}^{\rho}=\Omega_{3, k} \chi_{k}, \mathcal{G}_{k}=$ $\sum_{\text {if }}^{p} \lambda_{s=1} \lambda_{s, k} G_{s}, \mathcal{H}_{k}=\sum_{s=1}^{p} \lambda_{s, k} H_{s}$ and the inequality (??), the above inequality (??) is fulfilled

$$
\sum_{s=1}^{p} \lambda_{s, k}\left(\Xi+\Omega_{1, s}^{T} P \Omega_{1, s}+\Omega_{2, s}^{T} Z \Omega_{2, s}+\Omega_{3, s}^{T} W \Omega_{3, s}\right)<0,
$$

where $\Xi=\Xi_{1}+\Xi_{2}+\Xi_{3}$ being $\Xi_{1}, \Xi_{2}$ and $\Xi_{3}$ defined in (??) and (??). Note from Definition ?? and $\lambda_{s, k}=\lambda_{s}\left(t_{k}\right)$ the following properties: $0 \leq \lambda_{s}\left(t_{k}\right) \leq 1$ and $\sum_{s=1}^{p} \lambda_{s}\left(t_{k}\right)=1, t_{k}=k T_{s}, \forall k \geq 0$. Hence, the inequality (??) is true if the following LMIs hold, $\forall s=1, \ldots, p$ :

$$
\Xi+\Omega_{1, s}^{T} P \Omega_{1, s}+\Omega_{2, s}^{T} Z \Omega_{2, s}+\Omega_{3, s}^{T} W \Omega_{3, s}<0
$$

Finally, applying Schur Complement in $\Omega_{1, s}^{T} P \Omega_{1, s}, \Omega_{2, s}^{T} Z \Omega_{2, s}$ and $\Omega_{3, s}^{T} W \Omega_{3, s}$, it can be seen that (??) and (??) are equivalent, concluding the first part of the proof.

Stability of $S_{2}-\Delta_{2}$ : Proceeding in a similar way as (??), consider the inequality:

$$
\Delta_{k}^{V}+\left(y_{k}^{\xi}\right)^{T}\left(W \otimes I_{2}\right) y_{k}^{\xi}-\xi_{k}^{T}\left(W \otimes I_{2}\right) \xi_{k}<0,
$$

and the Lyapunov-Krasovskii function:

$$
\begin{aligned}
& V_{1, k}=e_{k}^{T}\left(P \otimes I_{2}\right) e_{k} \\
& V_{2, k}=\sum_{m=1}^{\tau_{1}} \beta^{2(m-1)} e_{k-m}^{T}\left(Q_{1} \otimes I_{2}\right) e_{k-m}+\sum_{m=1}^{\tau_{2}} \beta^{2(m-1)} e_{k-m}^{T}\left(Q_{2} \otimes I_{2}\right) e_{k-m} \\
& V_{3, k}=\tau_{1} \sum_{f=1}^{\tau_{1}} \sum_{m=1}^{f} \beta^{2(f-1)} \nu_{k-m}^{T}\left(Z_{1} \otimes I_{2}\right) \nu_{k-m}+\tau_{2} \sum_{f=1}^{\tau_{2}} \sum_{m=1}^{f} \beta^{2(f-1)} \nu_{k-m}^{T}\left(Z_{2} \otimes I_{2}\right) \nu_{k-m}
\end{aligned}
$$

where $\nu_{k}=e_{k+1}-e_{k}$. From (??) and following the same steps (??)-(??), we obtain the following conditions with $s=1, . ., p$ :

$$
\left(\Xi+\Omega_{1, s}^{T} P \Omega_{1, s}+\Omega_{2, s}^{T} Z \Omega_{2, s}+\Omega_{3, s}^{T} W \Omega_{3, s}\right) \otimes I_{2}<0
$$

By applying Lemma ??, we obtain that the above inequalities (??) are equivalent to:

$$
I_{2} \otimes\left(\Xi+\Omega_{1, s}^{T} P \Omega_{1, s}+\Omega_{2, s}^{T} Z \Omega_{2, s}+\Omega_{3, s}^{T} W \Omega_{3, s}\right)<0
$$

by pre- and post-multiplying by some permutation matrix $\mathcal{P}$. Finally, it can be seen that (??) is true if and only if (??) holds, which is equivalent to (??) by Schur Complement.

Hence, the fulfilment of LMIs (??) implies that subsystems $S_{1}-\Delta_{1}$ and subsystem $S_{1}-\Delta_{1}$ are robustly $\beta$-stable. Therefore, the overall system given in Lemma ?? is stable, taking into account that $\omega_{k}$ vanishes if subsystem $S_{1}-\Delta_{1}$ is stable (as discussed in Remark ??). Finally, the proof is concluded from the equivalence stated in Lemma ?? with the closed-loop system (??) and (??).

A feasible solution for a set of LMIs can be found by using numerical efficient and reliable algorithms based on convex optimization approaches (e.g., interior point [?]), which are available in standard commercial libraries (LMI Control Toolbox [?], SeDuMi [?], etc).

\section{Remark 8}

The design of $K, \gamma$ can be addressed by applying dichotomic search in Theorem ?? inside the range values $K>0$ and $0 \leq \gamma \leq 1$ with the objective of minimizing $\beta$ (maximum speed of convergence). 
Table I. Control designs under time-varying delays and the switching topology given in Fig. ?? (Simulation 1). First row, non-predictor. Second row: proposed weighted predictor and Third row: predictor with $\gamma=1$. Rightmost column: decay rate $\beta$

\begin{tabular}{|c|c|c|c|}
\hline Control method & $K$ & $\gamma$ & $\beta$ \\
\hline \hline no-predictor & $0.5 \cdot 10^{-3}$ & 0 & 0.9999 \\
\hline weighted predictor & $4.2 \cdot 10^{-3}$ & 0.61 & 0.9994 \\
\hline predictor & $3.6 \cdot 10^{-3}$ & 1 & 0.9995 \\
\hline
\end{tabular}

\section{SIMULATION RESULTS}

In this section, two comparative examples are provided in order to illustrate the effectiveness of the proposed weighted predictor-feedback formation control in the presence of time delays and timevarying switching communication topology.

\subsection{Simulation 1}

Consider a multiagent system (??) composed of $N=12$ agents. In the aim to show the benefits of the proposed weighted predictor-based control approach, the following three control strategies are given for comparative purposes:

- (i) Non-predictor feedback control: (??)-(??) $(\gamma=0)$.

- (ii) Weighted predictor-feedback control: (??)-(??).

- (iii) Predictor-feedback control (??)-(??) with $\gamma=1$.

Let the sampling period be $T_{s}=0.05 \mathrm{~s}$. The objective is to design the above controllers to reach the target formation depicted in Fig. ?? (solid blue line) as fast as possible. The non-predictor and predictor-feedback control (cases (i) and (iii)) are designed by applying Theorem ?? and dichotomic search in $K>0$ with $\gamma=0$ and $\gamma=1$ respectively in order to minimize the decay rate $\beta$. The weighted predictor-feedback controller parameters are designed with the same objective by means of Theorem ?? and dichotomic search (see Remark ??) in $K>0$ and $0 \leq \gamma \leq 1$. As a result, we obtain the control parameters given in Table ?? which ensure for each control design the $\beta$-stability of the formation control with $\beta$ given in the rightmost column of Table ??, for any time-varying delays satisfying $\tau_{1} \leq \tau_{j i, k} \leq \tau_{2}$ with $\tau_{1}=389, \tau_{2}=391$ and switching topology $\mathcal{A}_{\sigma(t)} \in\left[A_{1}, A_{2}, A_{3}\right]$, with $\left\{A_{1}, A_{2}, A_{3}\right\}$ given in Fig. ??.

Simulation results are depicted in Fig. ??, where the above control strategies (see Table ??) are compared in the presence of time-varying delays $340 \leq \tau_{j i, k} \leq 440$ (see rightmost side of Fig. ??) and switching topology $\mathcal{A}_{\sigma(t)} \in\left[A_{1}, A_{2}, A_{3}\right]$ with $\left\{A_{1}, A_{2}, A_{3}\right\}$ given in Fig. ??, and $\sigma(t)$ depicted in the leftmost side of Fig. ??. The left, middle and right parts of Fig. ?? show respectively the system response using non-predictor control design (given in Table ??, first row), the proposed weighted predictor-feedback design (given in Table ??, second row), and the predictor-feedback design with $\gamma=1$ (give in Table ??, third row). The upper part in Fig. ?? depicts the trajectories followed by each agent. The middle part represents the time evolution of the estimation of the rotation angle of the reference frame $\hat{\phi}_{i}$ made by each agent from (??), and the lower part gives the time evolution of the normalized cost functions $J_{1, k}$ (solid blue line) and $J_{2, k}$ (dashed red line), defined as

$$
J_{1, k}=\frac{\sum_{i=1}^{N}\left\|u_{i, k}\right\|^{2}}{\sum_{i=1}^{N}\left\|u_{i, 0}\right\|^{2}}, \quad J_{2, k}=\frac{\sum_{i=1}^{N}\left\|v_{i, k}\right\|^{2}}{\sum_{i=1}^{N}\left\|v_{i, 0}\right\|^{2}}
$$

where $u_{i, k}$ and $v_{i, k}$ are respectively defined in (??) and (??).

Note that in all three cases, the multiagent system is exponentially stable and the estimations $\hat{\phi}_{i}, i=1, \ldots, 12$ converge to the agreed rotation angle of the global reference frame $(\alpha \approx-2.4 \mathrm{rad})$. 

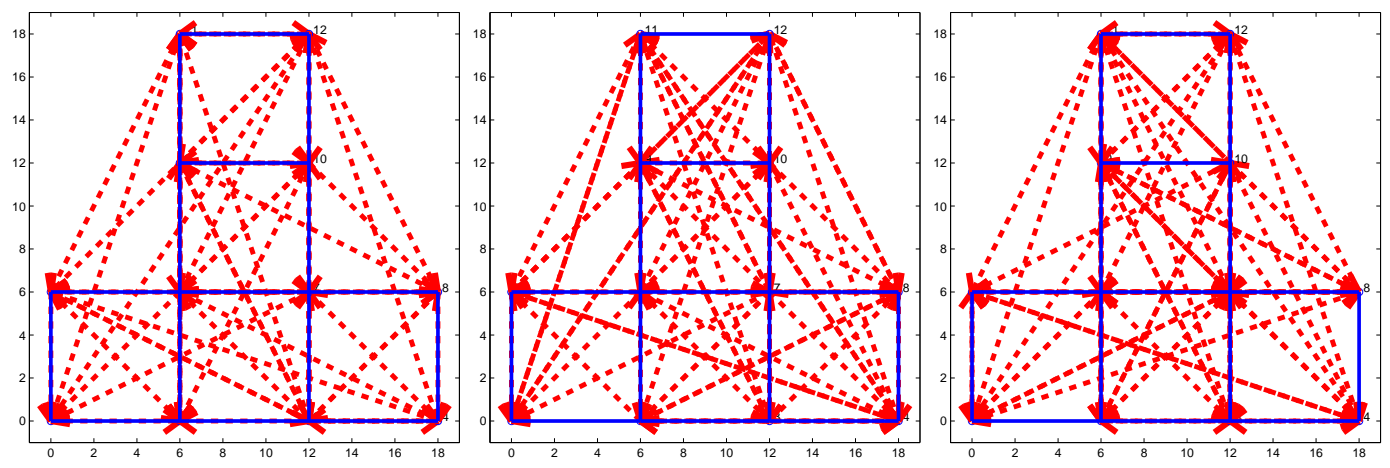

Figure 2. Target formation (solid blue lines) and switching communication topology (dashed red lines) with $A_{s}, s=1,2,3$ for Simulation 1 .
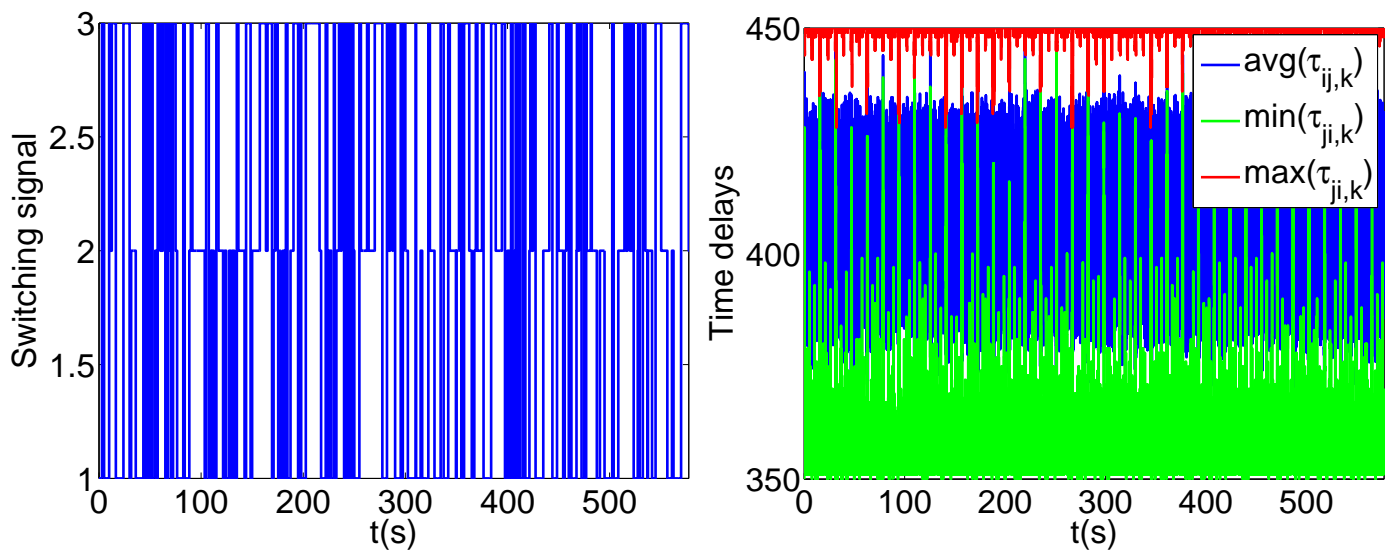

Figure 3. Left: Time-varying switching signal $\sigma(t) \in\{1,2,3\}$ for the communication topology $\mathcal{A}_{\sigma(t)} \in$ $\left\{A_{1}, A_{2}, A_{3}\right\}$ with $A_{i}, i=1,2,3$ given in Fig. ??. Right: time-varying delays (average value, minimum and maximum) for Simulation 1.

However, the minimum time required to reach the formation for this multiagent system with the given time-varying delay interval is around $600 \mathrm{~s}$ (see second and third row in left column, Fig. ??), being not possible to achieve faster convergence by using non-predictor control. However, by using predictor-feedback control with the designed controller parameters, the setting time is reduced to $100 s$ approximately (more than six times faster). It can also be appreciated how the trajectories followed by each agent are visibly improved using the proposed control scheme. Also, note that by choosing $\gamma=1$, the maximum convergence speed is a bit slower than using weighting predictor (around 150s), which reveals the effectiveness of delay compensation weighted by $\gamma=0.61$ in the sense of faster convergence. For a fair comparison, the same time-varying delay and time-varying switching topology patterns (given in Fig. ??) have been used to perform all simulations.

\subsection{Simulation 2}

Consider the same multiagent system as in the previous example, but defining a switching communication topology with lower degree of connectivity (see Fig ??, dashed red line). We proceed as in Simulation 1 with sampling period $T_{s}=0.05 \mathrm{~s}$, obtaining the control parameters given in Table ?? which ensure the $\beta$-stability of the formation control for any time-varying delays satisfying $\tau_{1} \leq \tau_{j i, k} \leq \tau_{2}$ with $\tau_{1}=34, \tau_{2}=36$ and switching topology $\mathcal{A}_{\sigma(t)} \in\left[A_{1}, A_{2}, A_{3}\right]$, with $\left\{A_{1}, A_{2}, A_{3}\right\}$ given in Fig. ??.

Simulation results are depicted in Fig. ??, where the control strategies given in Table ?? are compared under time-varying delays $30 \leq \tau_{j i, k} \leq 40$ given in the rightmost side of Fig. ??, and 

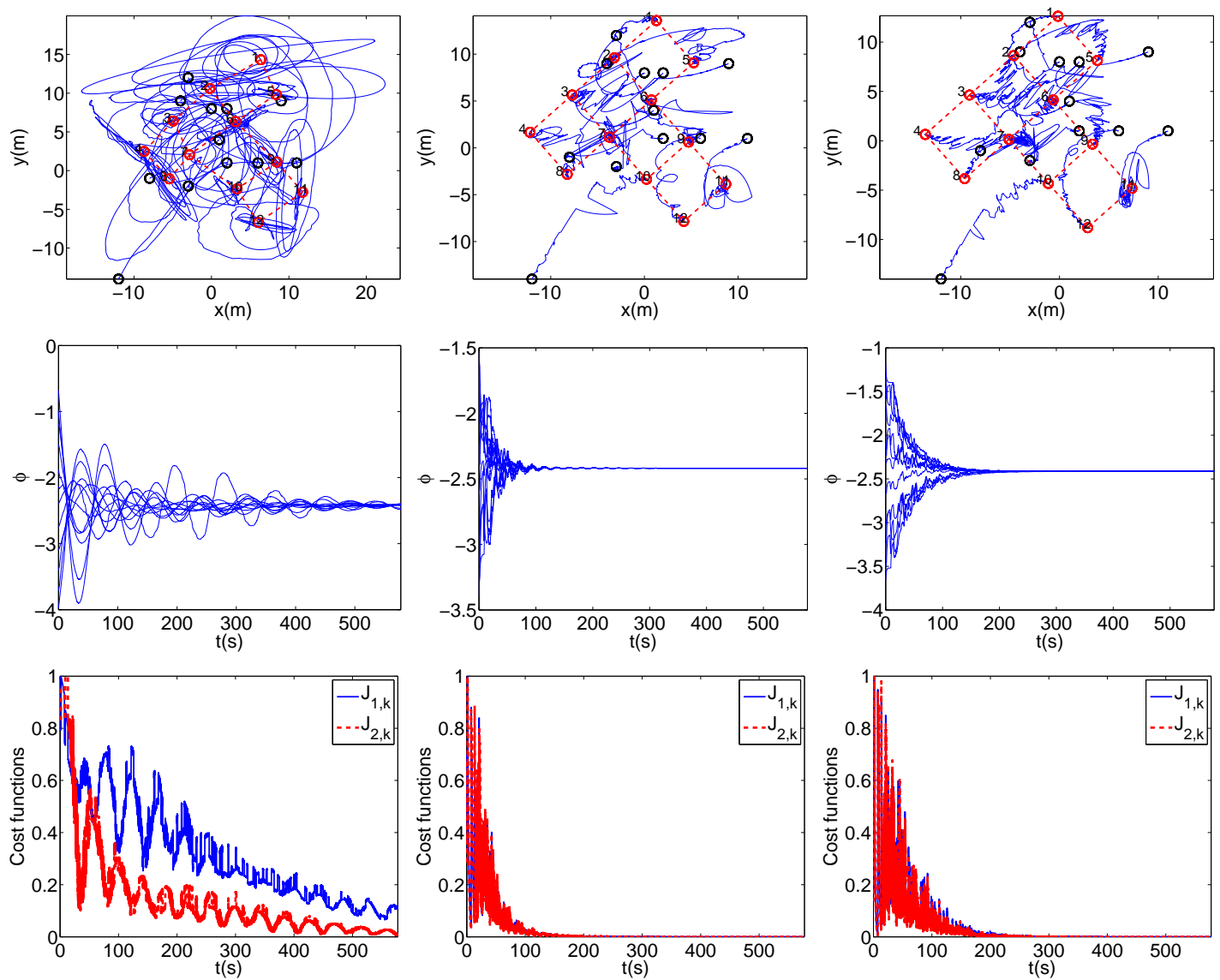

Figure 4. Comparative results between non-predictor (left), proposed weighted predictor (middle), and predictor control (right) given in Table ?? under time-varying delays $340 \leq \tau_{j i, k} \leq 440$ and switching topology $\mathcal{A}_{\sigma(t)} \in\left[A_{1}, A_{2}, A_{3}\right]$ given in Fig. ?? (Simulation 1). First row: trajectories followed by each agent. Second row: Estimation of the angle $\hat{\phi}_{i}$, and Third row: Normalized cost functions $J_{1, k}$ and $J_{2, k}$ in

Table II. Control designs under time-varying delays and switching topology given in Fig. ?? (Simulation 2). First row, non-predictor. Second row: proposed weighted predictor. Rightmost column: decay rate $\beta$

\begin{tabular}{|c|c|c|c|}
\hline Control method & $K$ & $\gamma$ & $\beta$ \\
\hline \hline no-predictor & $7.5 \cdot 10^{-3}$ & 0 & 0.9997 \\
\hline weighted predictor & $66.7 \cdot 10^{-3}$ & 0.28 & 0.9996 \\
\hline predictor & $56.2 \cdot 10^{-3}$ & 1 & 0.9999 \\
\hline
\end{tabular}

switching topology $\mathcal{A}_{\sigma(t)} \in\left[A_{1}, A_{2}, A_{3}\right]$ with $\sigma(t)$ depicted in the leftmost side of Fig. ??. In all three cases, the multiagent system is exponentially stable. However, it can be appreciated that by using the weighted predictor with $\gamma=0.28$, the faster response is achieved with a settling time less than $150 \mathrm{~s}$, whereas by setting $\gamma=1$, the formation control is not reached after $150 \mathrm{~s}$ due to slower convergence. Note that only a slight improvement can be appreciated with respect to non-predictor control $(\gamma=0)$ in comparison to Simulation 1. Hence, one can conclude that the effectiveness of delay compensation decreases as long as the degree of connectivity in the communication graph is lower. This fact means that the predictor-feedback term has less capability to counteract delays under weaker connected graphs since the prediction becomes less reliable, which is consistent with 

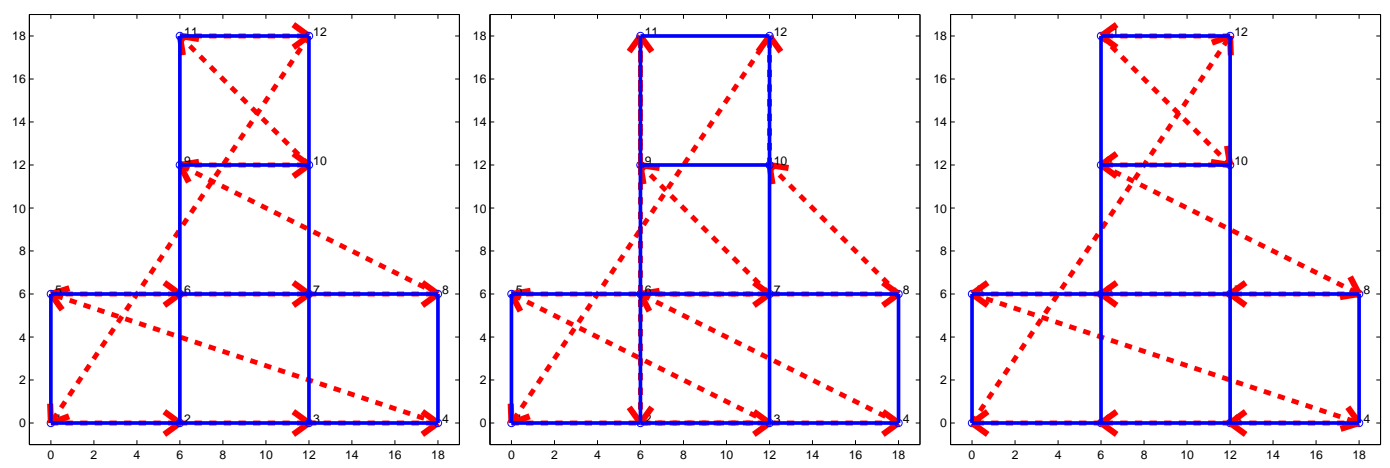

Figure 5. Target formation (solid blue lines) and switching communication topology with lower degree of connectivity (dashed red lines) with $A_{s}, s=1,2,3$ for Simulation 2.
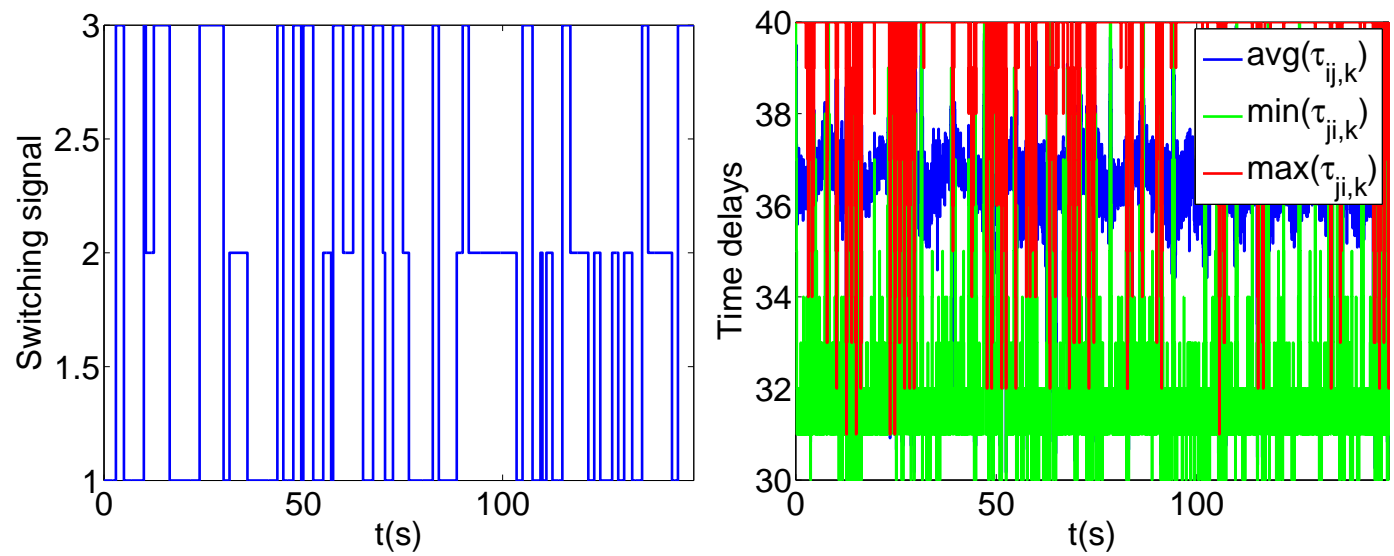

Figure 6. Left: Time-varying switching signal $\sigma(t) \in\{1,2,3\}$ for the communication topology $\mathcal{A}_{\sigma(t)} \in$ $\left\{A_{1}, A_{2}, A_{3}\right\}$ with $A_{i}, i=1,2,3$ given in Fig. ?? (Simulation 2). Right: time-varying delays (average value, minimum and maximum) for Simulation 2.

the design of a lower value for the weighting factor $\gamma$ for prediction terms in the proposed formation control: $\gamma=0.28$ in this case vs $\gamma=0.61$ in case of stronger connectivity (Simulation 1).

\section{CONCLUSIONS AND PERSPECTIVES}

We have presented in this paper a weighted predictor-feedback control strategy for systems with time-varying communication delays and switching communication topology. By properly designing the control gain and the weighting factor, better agents' trajectories and convergence ratios can be achieved in comparison to non-predictor feedback control, even in case of communication topology with low degree of connectivity. Future developments could address other relevant issues in formation control, such as guaranteeing the absence of collisions, or implementing event-triggered protocols with the objective of reducing the bandwidth usage and energy consumption.

\section{APPPENDIX}

\section{Theorem 2}

(Scaled Small Gain Theorem)[?] (Chapter 8) Given two subsystems $S$ and $\Delta$, the interconnected system $y=S e, e=\Delta y$ is robustly stable for any interconnected time-varying uncertain system $\Delta$ 

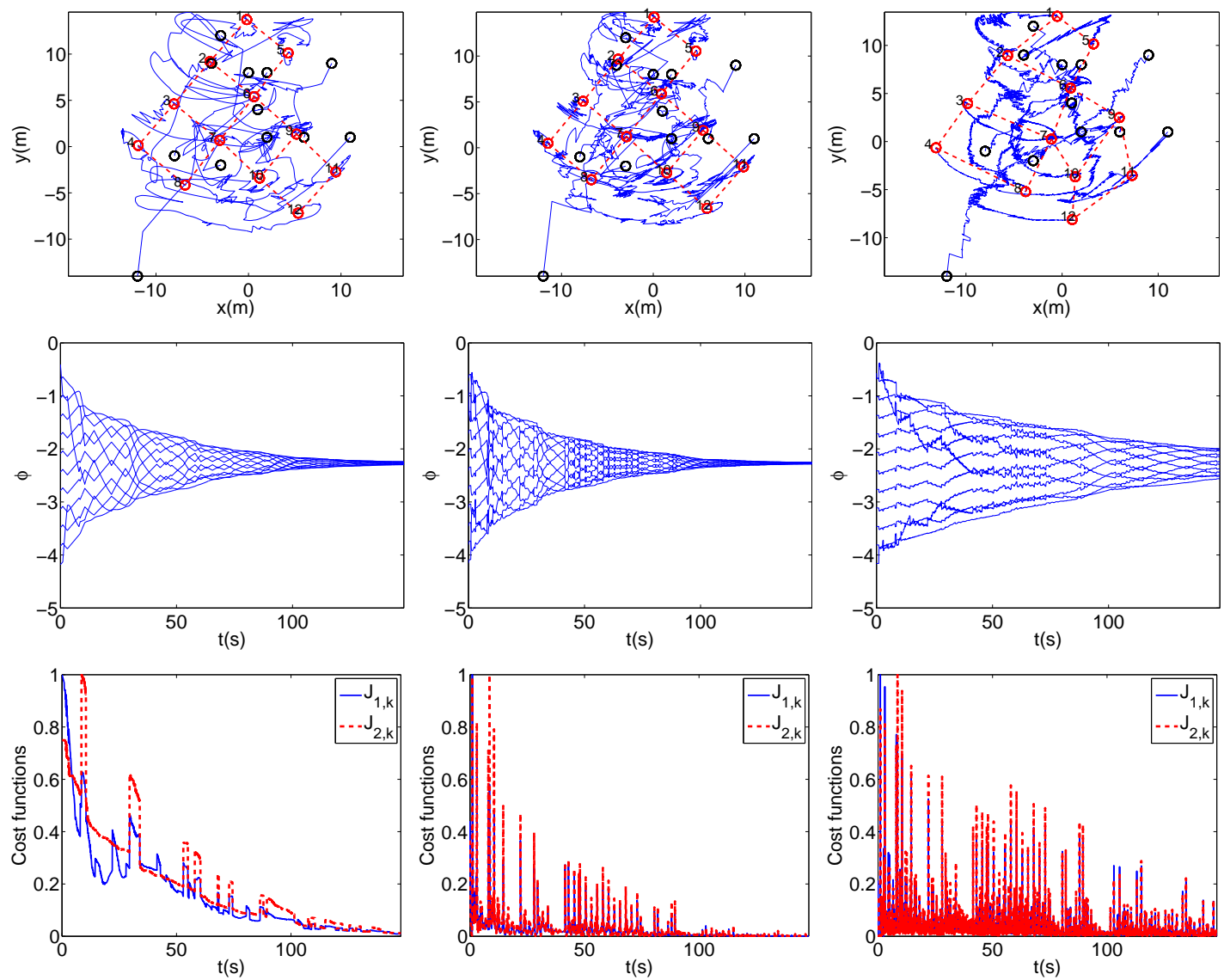

Figure 7. Comparative results between non-predictor (left), proposed weighted predictor (middle), and predictor control (right) given in Table ?? under time-varying delays $30 \leq \tau_{j i, k} \leq 40$ and switching topology $\mathcal{A}_{\sigma(t)} \in\left[A_{1}, A_{2}, A_{3}\right]$ given in Fig. ?? (Simulation 2). First row: trajectories followed by each agent. Second row: Estimation of the angle $\hat{\phi}_{i}$, and Third row: Normalized cost functions $J_{1, k}$ and $J_{2, k}$ in (??)

with $\|\Delta\|_{\infty} \leq 1$ if the following two conditions hold: (i) The system $S$ is internally stable and (ii) there exist a regular matrix $\mathcal{X}$ such that $\mathcal{X} \Delta=\Delta \mathcal{X}$ and $\left\|\mathcal{X} S \mathcal{X}^{-1}\right\|_{\infty}<1$.

\section{Lemma 2}

Given a discrete-time signal $\varepsilon_{k}$ and any arbitrary sequence of integer numbers $\tau_{k}$ satisfying $\tau_{1} \leq \tau_{k} \leq \tau_{2}, \forall k \geq 0$. Let $y_{k}^{\rho}=\varepsilon_{k+1}-\varepsilon_{k}$ and

$$
\rho_{k}=\frac{2}{\tau}\left(\varepsilon_{k-\tau_{k}}-\frac{1}{2}\left(\varepsilon_{k-\tau_{1}}+\varepsilon_{k-\tau_{2}}\right)\right),
$$

where $\tau=\tau_{2}-\tau_{1}$. Then, the time-varying operator $\Delta_{d}: y_{k}^{\rho} \rightarrow \rho_{k}$ renders $\rho_{k}=$ $\frac{1}{\tau} \sum_{i=k-\tau_{2}}^{k-\tau_{1}-1} f_{\rho}(i) y_{\rho, i}$, where

$$
f_{\rho}(i)= \begin{cases}1 & \text { if } i<k-\tau_{k}-1, \\ -1 & \text { otherwise }\end{cases}
$$

and satisfies $\left\|\Delta_{d}\right\|_{\infty} \leq 1$, where the symbol $\|.\|_{\infty}$ denotes the largest possible $\mathcal{L}_{2}$ induced norm of a general operator.

\section{Proof}

The proof is an adaptation of a similar result given in [?, Lemma 2] for continuous-time systems. 
Lemma 3

[?] Given two square matrices $A$ and $B$, the following equivalence holds:

$$
\mathcal{P}^{T}(A \otimes B) \mathcal{P}=B \otimes A
$$

where $\mathcal{P}$ is some regular permutation matrix.

\section{ACKNOWLEDGEMENTS}

This work was supported by projects COMMANDIA SOE2/P1/F0638 (Interreg Sudoe Programme, ERDF), PGC2018-098719-B-I00 (MCIU/ AEI/ FEDER, UE) and Fundación Universitaria Antonio Gargallo (project 2018/B004). 